\title{
(Murradambirra Dhangaang - Make Food Secure) Aboriginal Community and Stakeholder Perspectives on Food Insecurity in Urban and Regional Australia: A Qualitative Study
}

\section{Simone Sherriff}

The Sax Institute

Deanna Kalucy

The Sax Institute

Allison Tong

The University of Sydney

Nawazish Naqvi

University of Toronto

Janice Nixon

The Sax Institute

\section{Sandra Eades}

Curtin University

\section{Tangerene Ingram}

Riverina Medical and Dental Aboriginal Corporation

Kym Slater

Tharawal Aboriginal Corporation

Michelle Dickson

The University of Sydney

Amanda Lee

The University of Queensland

Sumithra Muthayya ( $\nabla$ sumithra.muthayya@saxinstitute.org.au )

The Sax Institute

\section{Research Article}

Keywords: Food insecurity, diet inequality, Aboriginal and Torres Strait Islander, Indigenous, stakeholders, food systems, chronic disease, urban, qualitative

Posted Date: May 5th, 2021 
DOl: https://doi.org/10.21203/rs.3.rs-463149/v1

License: (c) (i) This work is licensed under a Creative Commons Attribution 4.0 International License. Read Full License 


\section{Abstract \\ Background:}

Food insecurity affects one in five Aboriginal and Torres Strait Islander people residing in non-remote environments. Inequalities in diet contribute to the differential impact on diet-sensitive chronic diseases and the related burden of disease among Aboriginal and Torres Strait Islander people. This study aimed to describe Aboriginal community and stakeholder perspectives on food insecurity to get a better understanding of the key factors driving this issue and recommendations for potential solutions in urban and regional Aboriginal communities in Australia.

\section{Methods:}

Semi-structured interviews were conducted with 44 participants who were purposively selected. This included Aboriginal people in two communities and both Aboriginal and non-Aboriginal stakeholders from local food relief agencies, food suppliers, schools, and government. A conceptual framework was developed from food insecurity literature and we used the sensitizing concepts of availability, affordability, accessibility and acceptability or the lack thereof of healthy food to elicit interview responses. Interview transcripts were analysed thematically.

\section{Results:}

All participants felt strongly that food insecurity was a major problem experienced in the local Aboriginal communities. Five core areas impacting on food security identified: trapped in financial disadvantage; gaps in the local food system; limitations of non-Aboriginal food relief services; on-going impacts of colonization; and maintaining family, cultural and community commitments and responsibilities.

\section{Conclusions:}

This study found Aboriginal families in urban and regional Australia are experiencing food insecurity on a regular basis, which is impacted by a range of socio-economic, environmental, systemic and cultural factors, as reported by the participants. Our findings highlight the need to address system level changes in the food environment and acknowledge Aboriginal culture and food preferences while considering the development of programs to alleviate food insecurity among urban and regional communities.

\section{Background}

Food insecurity is a serious challenge experienced by Aboriginal and Torres Strait Islander people amidst a wider growing crisis of hunger and food inequality in Australia (1). As many as one in five Aboriginal and Torres Strait Islander people living in non-remote environments - who make up three-quarters of the total 
Aboriginal and Torres Strait Islander population in Australia -reported being food insecure in the national 2012-13 survey (1). This compares to one in four among remote Aboriginal and Torres Strait Islander communities and one in twenty-five among the wider Australian population. Hunger and food insecurity has been on the rise worldwide over the past many decades, and the economic fallout and food crisis resulting from the COVID-19 pandemic has further exacerbated this problem globally and in Australia (24). Food security exists "when all people, at all times, have physical and economic access to sufficient, safe and nutritious food to meet their dietary needs and food preferences for an active and healthy life" (5). Four key components - food availability, accessibility, acceptability and adequacy are highlighted in this definition of food security by the FAO.

Food insecurity can have significant lifelong consequences on the health and wellbeing of populations and, therefore, must be addressed as a significant public health priority by all levels of government. Mild to moderate food insecurity is associated with poor physical and mental health in both children and adults (6). Children are particularly vulnerable to both the short and longer-term effects of food insecurity, resulting in anaemia, greater learning and behavioural problems, mental health issues and increased risk of hospitalization, (7-9). Food insecurity is also directly related to higher rates of obesity and chronic disease in adults $(6,10)$. Food-insecure households tend to respond by adjusting the food budget, decreasing dietary variety and increasing the consumption of cheap, energy dense foods (11). National survey data show that about $95 \%$ of Aboriginal and Torres Strait Islander people (aged 15 years and over) report not eating adequate amounts of vegetables each day, with $41 \%$ of their energy intake derived from discretionary foods and drinks that tend to be high in added sugar, saturated fat, salt and/or alcohol (12), often as a result of unequal availability of, or access to, a healthy food supply $(13,14)$. This inequality in diet and food insecurity experienced by Aboriginal and Torres Strait Islander people contributes to the differential impact on diet-sensitive cardiovascular diseases and other chronic disease, and related burden of disease $(15,16)$.

Aboriginal and Torres Strait Islander people, who before European invasion and colonization had managed and sustained their food environment to meet their needs for over 60,000 years through a deep understanding of the land, water and connection to their Country (traditional lands), are now among the most socially and economically disadvantaged in Australia and experience challenges purchasing adequate quantities of food, as well as adequate quality healthy food due to financial and other constraints (17-20). Food environments and food insecurity experienced by Aboriginal and Torres Strait Islander people in non-remote Australia differ from those in remote areas $(21,22)$. While the urban food environment may offer a tremendous diversity of food for consumers, there are huge geographical disparities in access to fresh, healthy food (23). There is evidence from Australia and the United States that people living in lower socioeconomic (SES) areas often cannot afford to buy healthy food in their local neighbourhood, and incur additional costs to travel to shops further away where a greater variety of less expensive produce is available $(24,25)$. Another study in Australia found that people living in low-SES areas purchased a smaller variety of fruits and grocery foods that were low in fibre and high in fat, salt and sugar, compared to people in advantaged areas (26). In that study, SES was associated with food purchasing behaviours, suggesting that a decrease in food availability, accessibility, and affordability, 
made the purchase of some types of foods more difficult in disadvantaged areas. Greater exposure to unhealthy foods and pervasive marketing of these foods in low-SES areas add to the complex, urban obesogenic food environment (27-30).

There has been limited research examining the key drivers of food insecurity among Aboriginal and Torres Strait Islander people living in non-remote areas and it is, therefore, important to gain a complete understanding of the issue and underlying systemic and other factors prior to attempting to develop and implement solutions (31). Developing this requires an understanding and recognition that food security is very much a complex and dynamic issue at the intersection between social and cultural, economic, agricultural, nutrition and health sectors $(20,32)$. Combining holistic Indigenous knowledge and addressing systemic factors across these multiple sectors $(33,34)$ can complement this approach to understanding food insecurity. Therefore, this study conducted in urban and regional New South Wales (NSW) aimed to describe the perspectives, knowledge and beliefs of Aboriginal families, Aboriginal Elders and Aboriginal staff members linked to two Aboriginal Community Controlled Health Services (ACCHSs) in New South Wales (NSW) (35), and that of a range of stakeholders in government and other organizations linked to the local food system, on the key drivers of food insecurity and their recommendations for potential solutions. Much needed data on gaps in our understanding of this problem can provide a better awareness of how to support Aboriginal communities experiencing food insecurity and guide the development of community-led programs to strengthen local food systems.

\section{Methods}

This research used a qualitative approach and was informed by Indigenous Standpoint Theory (36). By using this approach, we attempted to stay as close as possible to the voices of the participants and allowed their perspectives to form the findings. This process of qualitative data collection and analysis was particularly appropriate to explore this under-investigated research area. For this paper we respectfully use the term Aboriginal hereafter to refer to the traditional custodians of lands in NSW, in line with current recommendations (37).

\section{Setting}

The research was conducted in two communities in NSW: Campbelltown, an urban outer suburb of Greater Sydney with a population of 78,849 people (4.7\% Aboriginal) (38); and Wagga Wagga, a large regional area located in inland NSW approximately five hours' drive from Sydney which has a population of 62,385 people (5.6\% Aboriginal) (38). Aboriginal communities have a lower weekly household income of $\$ 105$ in Campbelltown and $\$ 242$ in Wagga Wagga when compared to the median weekly household income for their respective Local Government Areas (39).

\section{Participant selection and recruitment}

Ethics approval for this study was provided by the Aboriginal Health and Medical Research Council of NSW (1226/16). 
This study is embedded within the Study of Environment on Aboriginal Resilience and Child Health (SEARCH) $(40,41)$. SEARCH is a large cohort study that has been co-created with four ACCHSs in NSW to assess the health and wellbeing of 1669 Aboriginal children and their families living in urban communities in NSW to inform delivery of relevant programs and services (42). Food and nutrition insecurity were reported by parents/caregivers in SEARCH to be the third most significant factor affecting the health and wellbeing of their children, after "loving family relationships" and "culturally competent healthcare" (43).

Participants for this study were recruited through two of the partner ACCHSs in SEARCH: Tharawal Aboriginal Corporation located in Campbelltown, and Riverina Medical and Dental Aboriginal Corporation in Wagga Wagga. We used purposive sampling to include a range of Aboriginal community members, including Aboriginal Elders and families, ACCHS board members and ACCHS staff, and Aboriginal and non-Aboriginal representatives from food relief agencies, schools, local government, council, Local Health Districts, Primary Health Networks, and food suppliers. ACCHS staff identified local Aboriginal community members and other stakeholders who could offer insights about food insecurity in the local Aboriginal community, and a list of potential participants was developed. Participants were recruited via telephone or in person by an ACCHS or SEARCH staff member and were advised that the study was being conducted to gather Aboriginal community and stakeholders' perspectives on food insecurity faced by the local Aboriginal community.

\section{Data collection}

An interview guide (Supplementary file 1) was developed by SM and AL based on available literature and definition of food security described above and refined through discussions with the research team and community partners who have expertise in Aboriginal health, public health nutrition, qualitative research and epidemiology. A conceptual framework was developed by reviewing the literature on food insecurity and we used the sensitizing concepts (44) of availability, affordability, accessibility and acceptability or the lack thereof healthy food to develop our interview guides and inform our analyses $(5,45)$. Questions focused on participants' knowledge and beliefs around food insecurity, challenges, and barriers to food security for themselves and for the local Aboriginal community, factors that influence it, and ideas for actions to improve the local food system.

Semi-structured interviews were conducted between September 2017 to August 2018. They were conducted either face-to-face at the local ACCHSs, at a location convenient for the participant, or over the telephone by four researchers (SS, SM, DK and JN), two of whom were Aboriginal researchers (SS and $\mathrm{JN}$ ). All interviews had an Aboriginal researcher (SS or JN) conducting the questioning together with another researcher (SM or DK) to ensure cultural safety and the accurate interpretation of participant responses. Participation was voluntary and written, informed consent was provided prior to the interview being conducted. Recruitment ceased when data saturation was reached, where interviews were no longer giving additional information to develop new themes but instead were endorsing already identified themes (46). All interviews were audio-recorded and transcribed verbatim, with participant permission.

\section{Data analysis}


Two authors (SS and NN) independently read all transcripts and two additional authors (SM and DK) independently read half of the transcripts each, and all developed preliminary themes relating to food security. Thematic analysis was used drawing on principles of grounded theory to guide the analytic process (47). Four authors (SS, DK, SM, NN) coded the transcripts line by line to inductively identify emerging themes. The researchers (SS, DK, NN, SM) met regularly to refine themes and sub-themes. Each theme was developed with data from the transcripts by SS, DK, SM and NN, and then further refined through analytical discussions with AT. HyperRESEARCH, version 4.0.1 (Research-ware Inc., Randolph, MA, USA) was used to code the data (SS). Findings are reported using Sandelowski's qualitative description approach (48), whereby data are represented in a way that most stakeholders would agree is acceptable and to stay as near to the data by summarising it in participants' everyday terms (48).

The COnsolidated criteria for Reporting Qualitative Research (COREQ) framework was used to report this study (49).

\section{Results}

Of the 52 people who were invited to participate, 44 (85\%) agreed and completed the interview. The duration of interviews was on average 30 minutes. Participant characteristics are described in Table 1. Non-participation was due to scheduling conflicts, illness, and non-response.

\section{Table 1: Participant characteristics $(n=44)$}




\begin{tabular}{|c|c|c|}
\hline Characteristics & $n$ & $\%$ \\
\hline \multicolumn{3}{|l|}{ Gender } \\
\hline Male & 12 & $27 \%$ \\
\hline Female & 32 & $73 \%$ \\
\hline \multicolumn{3}{|l|}{ Aboriginal status } \\
\hline Aboriginal & 24 & $50 \%$ \\
\hline Non-Aboriginal & 24 & $50 \%$ \\
\hline \multicolumn{3}{|l|}{ Age (years) } \\
\hline $20-39$ & 15 & $34 \%$ \\
\hline $40-49$ & 10 & $23 \%$ \\
\hline $50-59$ & 8 & $18 \%$ \\
\hline $60-69$ & 6 & $14 \%$ \\
\hline $70+$ & 5 & $11 \%$ \\
\hline \multicolumn{3}{|l|}{ Highest level of Education } \\
\hline Masters or above & 6 & $14 \%$ \\
\hline Bachelor's degree & 8 & $18 \%$ \\
\hline Diploma & 6 & $14 \%$ \\
\hline Certificate/some College & 5 & $11 \%$ \\
\hline High school & 15 & $34 \%$ \\
\hline Primary school & 1 & $2 \%$ \\
\hline No answer & 3 & $7 \%$ \\
\hline \multicolumn{3}{|l|}{ Stakeholder group } \\
\hline ACCHS staff and board members & 9 & $20 \%$ \\
\hline Aboriginal community members (Elders, parents, and caregivers) & 13 & $30 \%$ \\
\hline Food supplier, food relief organizations/charities & 12 & $27 \%$ \\
\hline Council, local government, LHD and Education & 10 & $23 \%$ \\
\hline
\end{tabular}

ACCHS =Aboriginal Community Controlled Health Service

LHD $=$ Local Health District 
We identified five core themes: trapped in financial disadvantage, gaps in the local food system, limitations of non-Aboriginal food relief organizations, on-going impacts of colonization, and maintaining family, cultural and community commitments and responsibilities. An artwork of how these concepts tell a story of food insecurity in the two Aboriginal communities is shown in Figure 1. The respective subthemes are included in the descriptions below.

\section{Theme 1: Trapped in financial disadvantage}

\section{Caught in constant debt and struggling to survive}

Participants felt families were running out of money before each pay cycle and were unable to purchase food. They noted this was due to high rates of unemployment and low income, often leading them to borrow money from family or friends and take high interest loans to support their household. This made it more difficult for families to pay for necessities including food as families:

"...think that the best thing to do is go and get one of them daily loans to have money, then they're paying back triple the amount which leads them into debt. Then they can't buy anything...it's just a circle that just keeps going around and around". (Aboriginal community member)

"I think they cope because it's family-orientated you see, and they go to their families if they run out of anything. If you run out of sugar - your neighbour comes along for sugar you give it to them...it works better that way". (Aboriginal Elder)

These problems were intensified for single parent or single income families who felt they were struggling to survive:

"I would like to give the kids more fruit and veggies but you can't on a budget, as the healthy stuff is always priced a lot higher than unhealthy stuff. That's why everyone just sticks to the unhealthy stuff". (Aboriginal mother)

\section{Rationing and relying on cheap, filling foods}

Participants described rationing food to ensure there was enough to go around until more money or food could be sourced. This included parents/caregivers eating less or skipping meals for up to a few days at a time to ensure their children had enough food. Other strategies, such as attending playgroups and other community events that provide food were used to ensure their children have a meal that day.

Participants explained that families felt resigned to choosing cheap, unhealthy foods that "clog the kids up" just to provide enough food for their families to survive and consequently this made them feel disheartened:

"I had one mum who came in and said to me, I have five kids and yes I know I don't eat properly but if I buy myself a large hot chips and a loaf of white bread, my kids are all full and can go to bed happy and satisfied. You're telling me to eat more fresh fruit and vegetables I don't even know if they're going to eat it. 
I can't afford to buy it. I don't even know if I'm going to waste my money on it. I know that's what's better for them but at the end of the day I'm happy when they go to bed with their tummy full". (ACCHS staff member)

Participants felt families in their communities were generally big meat eaters but noted that good cuts of meat are often expensive and unaffordable for many family budgets. This leaves many to purchase cheap meat packs predominately filled with sausages, mince and cheaper processed meats:

"If you've got eight people in your family, you're not going to go and buy eight T-bone steaks. You're going to buy a bucket load of rissoles or sausages to feed that family. I think that's what it comes down to". (Aboriginal community member)

\section{Dominating and competing priorities draining the food budget}

Participants explained that many Aboriginal families in their community struggled just to cover essential, unmovable expenses (i.e. rent, childcare, other bills) and may prioritise financial resources towards fulfilling those essentials, which can deplete the food budget. As an Aboriginal LHD staff member said:

"...you're going to have higher rentals, lack of accommodation, so food becomes last... if it's the end of a pay packet".

Participants described that some families worried about high utility costs associated with cooking food at home, and therefore chose quick cooking meal options that were often unhealthy.

Some participants felt that money used for tobacco, drugs, alcohol and gambling also put pressure on the family food budget and, therefore, was a major concern within their community:

"...there's probably some other issues that might affect that [food budget] and that could be smoking, so cigarettes are quite expensive, gambling, and maybe drugs and alcohol". (Aboriginal community member)

"...it's hidden, un-talked about. I think gambling has the biggest impact within our Aboriginal [community], and it's not addressed". (Aboriginal LHD staff member)

Some participants spoke about how families felt pressure to keep up appearances with the latest trends in order to avoid judgement and do not want to be seen as 'poor' or 'struggling', which they felt resulted in some families purchasing items such as the latest televisions, gaming devices, mobile phones and iPads that put a strain on the budget.

\section{Theme 2: Gaps in the local food system}

\section{Lack of healthy options and demotivation stemming from insufficient investment}

Participants described the unavailability of healthy food options both in terms of takeaway shops and supermarkets in the suburbs where most Aboriginal families live. Participants felt that having only smaller independent supermarkets nearby meant that healthy food is often stale, limited, and expensive. As an 
Aboriginal Elder explained "well you go to the supermarket, and it's disgraceful. There's nothing there. There's nothing fresh. It's mostly all tinned stuff". It was noted that while healthy food is more available at larger supermarkets in other suburbs, it is still unaffordable for many families:

"...no one can afford to buy it, because the healthy food is so expensive. It's a big factor, like everyone wants all these kids to eat healthy, but they're not looking at the price of things". (Aboriginal community member)

Participants also described how many local shops had closed in the suburbs where most Aboriginal families live and that there had been a lack of ongoing commitment from their local councils to ensure adequate infrastructure in their communities. As an ACCHS staff member explained, "lots of shops have closed in our suburb and there is no commitment from council, like you don't think people are worth investing into". They felt this affected morale as:

"... we've been slumped as the crappiest suburb in the city. But they're not helping. What are the people going to do against that? They are going - alright you don't want to help us. We are not going to help ourselves". (Aboriginal community member)

"... it really puts a bad community vibe and spirit. Like you don't think people are worth it- and it's a tactic used by the council, the Department of Housing to people won't want to stay if we don't make it a nice environment". (Aboriginal LHD staff member)

Participants also spoke about how their suburbs are often looked down upon by the wider community, as one Aboriginal family member explained, "this place is the greatest place I have ever lived in... I get angry because of the way it's trashed out by people. They have the wrong perspective of it".

\section{Abundance and easy access to unhealthy fast food}

Participants described how families are surrounded by unhealthy convenience foods in their suburbs, as an Aboriginal Elder described there is "the fish and chip shop, and the Domino's pizza - the other pizza up the road. Now that we've got McDonalds, a lot of them will walk down to McDonalds". As well as being more available, participants felt unhealthy takeaway options were more convenient "you just ring and order hot chips, that's it, go in the shop for fresh bread, then go and pick up the chips on the way out and there's lunch and it feeds them all", said an ACCHS staff member. Another participant explained:

"... at Dominos you can get pizzas for $\$ 6$ certain ones and they [children] like those ones. So we can get five or six pizzas and it's cooked and it's about the price of what we would have spent on dinner if we're preparing it all". (Aboriginal community member)

Some participants felt that the abundance of fast-food options in their suburb, combined with the view that they are just as cost effective as home cooked meals drives many families to overwhelmingly choose fast food.

\section{Lack of stable housing available and cooking facilities}


Participants noted that many families do not have access to safe and stable housing, instead having to stay:

“...in a car, somebody else's place, or you couch surf. Under somebody's house. Like it's all of those sorts of things are still in play today". (Aboriginal LHD staff member)

They felt not having safe and stable housing leaves these community members without access to basic cooking and food storage facilities such as a fridge or a freezer and is a major barrier to being able to prepare healthy foods:

"... most of our clients are in Housing. You get the ones that their fridge is broken but don't know that they could get a new fridge pretty fast. Instead they stay without one for months. You get the ones that don't buy food because they might have an infestation of cockroaches and - or their oven's broken, don't have a microwave". (ACCHS staff member)

Participants also explained that many families live in older social housing residences which have inadequate storage and bench space in kitchens making it difficult to prepare and store foods from a weekly shop for the family: as an Aboriginal stakeholder explained, "I've got a kitchen maybe half the size of this table". They felt the lack of storage meant families were spending more on food as they were doing daily shops, more likely to do more impulse shopping, and were driven towards purchasing easy, take away options.

\section{Limited transport options to access healthy food shops and large supermarkets}

Participants described how the lack of affordable, fresh food in their area forces Aboriginal families to travel to the town centre to access the larger supermarkets that have a wider variety of produce and cheaper options. They felt this can be difficult for families without cars or money to run their car, as there was limited public transport in their area and the cost of the bus or a taxi for families on a low income was taking money away from the food budget. Participants explained how limited public transport options in the local area means some people are having to walk home with their groceries, which limits their ability to do a large shopping trip. On the contrary, some non-Aboriginal participants felt there were adequate, affordable public transport options for the community to be able to access food shops and supermarkets.

Participants also noted that:

"... if there's no buses running at that time, and they can't get a lift off someone, then they'd have to walk or catch a taxi... on a Sunday when there's no buses running, that would be difficult". (Aboriginal Elder)

Participants explained how some families who have their own transport can go to several different grocery shops to get the cheapest deals, as an Aboriginal community member described "I've got an app on my phone that - I check it before I go shopping" to look through local catalogues to seek out the best deals on food. 
Participants also spoke about how racism plays a role in limiting transport options for families, who struggle to get a taxi to pick them up from their suburb as taxi drivers "often won't come to our suburb, unless they know you" (Aboriginal LHD staff member). Participants felt many families have faced racism on public buses when having to travel with their children and groceries and thought these negative experiences put some people off utilising public transport. Participants described that families who experience mental health concerns had additional challenges in accessing public transport to food shops:

"... a lot of people including me with mental health and all that you wouldn't be comfortable getting on a bus. I have that much anxiety about getting lost or climbing on the wrong bus or seeing someone and sitting there either not wanting to talk to them or being scared of someone if you see someone. I know it sounds stupid but that would be an issue for heaps of people". (Aboriginal mother)

Participants felt utilising public transport for food shopping was challenging for single parents who often needed to bring along their children which added additional stress and limitations:

"...it's hard enough for us to shop without the kids let alone having to go and shop with your kids, take them all on the bus and then have all your shopping. Then once you've got your youngest one out of the pram how do you carry it all? You have to push the trolley then if you're too far away from the shops it's too hard". (Aboriginal mother)

\section{Theme 3: Limitations of non-Aboriginal food relief services}

\section{Reliance on food relief and the inflexibility of services}

Participants felt many families in their community relied on emergency food relief services and subsidised food boxes:

"...they've got Vinnies (St Vincent de Paul Society) and they've got plenty of places where you can go up and get a food order and they won't knock you back. They give you good substantial stuff. It's always meat and veggies and things like that". (Aboriginal Elder)

Participants also described the fluctuating nature of food insecurity, where people may lose employment and experience food insecurity for a period of time. As explained by an Aboriginal Elder, "a lot of people are newly finding themselves in these situations that don't know how to access [emergency food relief]". However, some participants who were employed considered themselves to be the "working poor", making them ineligible for many food relief services. As an Aboriginal mother described, "I run out of food, and I work. So, I don't have a healthcare card. I can't go to St Vincent de Paul and go get a hamper or something like that".

Participants explained that Aboriginal families are generally larger and felt some food relief organizations do not take this into consideration. A participant explained how they felt short changed:

“...\$30 for me and six children... for the single families with two kids it was $\$ 20$. I don't know how they got a $\$ 10$ difference with an extra four kids. But that's how they work it out". (Aboriginal mother) 
Another Aboriginal mother spoke about having to "wait a week for that appointment" to get a food voucher, and while some participants acknowledged a week may not seem like a long time, they also noted the challenges of trying to survive for this period with no money or food. One Aboriginal mother described that "there is no compassion, nothing. No compassion at all" when accessing these services for help. Participants also felt the restrictions on food relief services such as time limitations on how regularly you could access food vouchers or hampers made it difficult. As another Aboriginal mother described, "I don't want to sound bad or anything like that. I'm appreciative of this...but you cannot get no other help for three months. Three to six months or something like that".

\section{Stigma and shame linked to accessing non-Aboriginal food relief services}

Participants described that Aboriginal people are proud people and felt that many families in their community would not be comfortable receiving free items such as food hampers as "we don't want to be looked on as poor or judged" suggesting that it makes people "feel shame as they don't want to be seen as a charity case", an Aboriginal Elder explained.

Some participants reported feeling judged by food relief agencies and expressed that some of them are either unable or unwilling to try and better understand the Aboriginal community's needs. They also felt that some families are far less likely to utilise these services due to the feeling of shame: "they've got that shame around going down anywhere to get food or anything" an ACCHS staff member explained. Participants described that some families are "too shame [sic] to say we can't get enough food because we are overweight", consequently they did not feel worthy to go and access food relief services.

Participants explained that food hampers provided by local charities are often made up of food which is no longer fit for sale. For example, an Aboriginal community member explained how the food is, "clearance stuff from Woolworths. They go to Woolworths and they get the things that they're going to throw out. They make them into hampers and then go to disadvantaged communities". They described how knowing this makes some families feel disheartened about receiving this food as "a lot of their stuff is out of date... if you've got no food, what's your option?".

Participants discussed how some Aboriginal families are reluctant to access food relief services for food hampers or vouchers as:

"...people have experienced families and kids being taken away so there is a fear of accessing services as they think you can't provide for your kids so they might get taken way". (Aboriginal LHD staff member)

Consequently, participants believed that some parents/caregivers would rather seek assistance from other family and community members than from local agencies, which further added to the financial burden experienced within the community.

Theme 4: On-going impacts of colonization

Experiences of trauma and disruption to family structures

Page $14 / 31$ 
Participants spoke about the high rates of Aboriginal children being taken from their families, both in the past, during the Stolen Generations period where there were policies for Australian federal and state government agencies, church missions and welfare bodies to forcibly remove Aboriginal children to be raised in institutions and by white families (50), and currently through removal of children into the 'out-ofhome' care system with non-relatives (51). They felt this has led to intergenerational trauma and disrupted family structures:

"...the interruption to our parenting practices due to trauma and stress, and the impact, because when you're stressed and trying to feed your family, it is easier to get the two-dollar chips and that". (Aboriginal Elder)

Participants also described racism, frequent community deaths, high rates of youth suicide and harassment from police causing constant stress. They felt this impacts on motivation to purchase and cook healthy food and leads families to turn to easier meal options as:

"So if we're struggling, automatically, you're going to go to a fast food shop. So if that's happening maybe two or three times a week within the family, you can guarantee we're going to face obesity, we're going to have diabetes. It's just stress, it's mental health on top of that, yeah. It just affects that whole cycle. That's from the top of our head right down to our toes". (ACCHS staff member)

Healthy eating and food affordability were also described as a challenge for parents/caregivers who were suffering with social and emotional wellbeing difficulties themselves or had children, or multiple family members experiencing inter-generational trauma. An Aboriginal community member explained that families are overwhelmed and stressed which means they are:

"...focussed on getting the task done [cooking] and that, I wasn't conveying the information or teaching them that. My whole life was about get this over and done with. You know like having time for that and stress, and I just wanted to escape everything". (Aboriginal community member)

\section{Cultural identity and food preferences}

Participants spoke about how colonization and food rations (such as rice, flour, sugar, tea and fatty meats (52)) received during the mission era (refer to footnote) drastically changed peoples diets, and still impacted on food choices today amongst Aboriginal families in their community (53). They felt that many foods such as damper (made with white flour), devon (processed luncheon sausage), white bread and hot potato chips are now seen as 'Aboriginal cultural foods':

"...getting in the mindset of our mob to understand, because we very much see this as cultural food. You know like our devon and potato chips. You know we do think of that like that, but why, and understanding why our diet has changed". (Aboriginal LHD staff member)

Participants also explained that their communities were very protective of maintaining these foods and pushing back against modifying them to make them healthier: 
"...even with the Elders. So in the kitchen we make just your normal damper and just went, oh how about this week we try a half wholemeal and half white flour. It was oh, if looks could kill I would have been dead that day". (ACCHS staff member)

"...the [ACCHS] had a healthy food policy, and people were so resistant because they thought we were taking away their culture and their practices by saying now you've got to be like white fellas and eat this good way. We were forced like this because of those colonised practices". (ACCHS staff member)

Aboriginal community members thought any initiatives that organizations deliver around healthy eating and lifestyle choices should ensure culture is always at the centre underpinning all the activities in their community:

"We are like this because of those colonised practices. I think that any programs or things like that, we need to do that education and bringing back the cultural part of it". (Aboriginal LHD staff member)

\section{Effects of inter-generational poverty on food choices}

Participants described how growing up in poverty has made many parents want to ensure their own children have more than they had, which leads them to purchase perceived 'luxury' foods they were not able to have as a child, such as soft drinks and takeaway meals:

"I looked in my cousin's fridge, when I came over once... and I thought oh my God, all these kids have got is water to drink. Those poor little things. I still have that image but now in my head thinking oh my God, my kids were the poor kids. My kids were the ones who were fed the poor nutrition and given that [fast food], because I believed this way. I can remember thinking I should chuck her [my cousin] some more money. (Aboriginal LHD staff member)

"... you see a lot of Aboriginal people, they grew up really, really poor, now that they've grown up and they've got the money, a lot of them are overweight. Because they can afford that good [luxury] food". (Aboriginal LHD staff member)

Participants described how inter-generational poverty has instilled the need to purchase cheap and often unhealthy foods to ration them around the family to survive:

"When I look back on my life I think about it and I think, was I actually ever hungry? Like we were poor and that, but did I actually go starving and without food. I'm thinking, no but I lived as if I was a starving child because my father was starving, his grandmother was starving, and food security was in our mindset". (Aboriginal LHD staff member)

\section{Generational loss of healthy food knowledge and preparation skills}

Aboriginal Elders spoke about how they have observed a generational gap in knowledge in the younger generation in their community about how to cook cheap, quick and healthy meals, which they felt was owing largely to children being removed and experiencing associated trauma: 
"...you know that generational slippage when there is trauma or interrupted parenting practices, kids removed, coming back in, this is stuff [healthy meal preparation] we learnt but now how are we passing that and teaching that on?". (Aboriginal Elder)

"...it's the young mums, you need to target the young mums, because all of a sudden, they've got a baby. It's all well and good when they've giving them the formula and then the baby food, but then: okay, it's time to start eating real food, what do you feed the baby?"(Aboriginal Elder)

Participants felt that some parents/caregivers lack knowledge about healthy foods and how to cook them, as an ACCHS staff member explained, "I know kids that don't even eat fruit and veg. It's sad that the parents aren't instilling that into their kids from a young age". Participants also noted that parents/caregivers may not have skills in how to budget, prepare for meals or to write up a weekly shopping list. This gap in budgeting and meal preparation skills was thought to cause many families in the community to spend more money as they are shopping daily:

"... if families are taught meal prepping, young families, that you can have these meals in your freezer, whether you have two the next week or something, or it's lunch the next day or dinner the next night. It's something that young people need to be taught". (Aboriginal Elder)

Overall, participants thought that group education led by the Aboriginal community would help build skills in young families to budget, prepare for and cook healthy meals and help to ensure these skills would be passed on to future generations:

"Well, I think the community have to get off their bum too and think about what can we do to make a better community... We need to think about things that are not too difficult for people to take on together. Even if it's just starting a little garden and then it grows. You remember that song, from little things big things grow". (Aboriginal Elder)

"I think it [programs] would be better in groups and then they lift each other up, rather than just going by yourself, it's a lonely world. I think we need to do that by community groups". (Aboriginal Elder)

\section{Theme 5: Maintaining family, cultural and community commitments and responsibilities}

\section{Sharing resources amongst family and community}

Participants discussed the unique kinship networks with extended family that exist in Aboriginal cultures. They explained this means families are often providing food and resources not just to people within their household but amongst their wider kinship and community networks, which is not typical amongst nonAboriginal people:

"... sometimes there'd be about 17 plates I'll dish out because I'm feeding for me and my family, my sisters, my nephews, my niece, other kids that come there and stay with us". (Aboriginal community member) 
"I've had heaps of people come and live with me because they've got nowhere to live, and they're not on Centrelink or anything like that so they can't help contribute. But you've still got to feed that extra mouth sort of thing. You can't turn them away. So you and your family are struggling as well as the other person really. So I think that's the major thing, is taking on board more people than you can afford to feed sort of thing". (ACCHS staff member)

Sharing of food and resources was frequently described as a positive way to manage food insecurity within the community:

"... because of the way Aboriginal families support each other - if one household has run out of food, then they can go and see another family member who can assist them, whether they give them food, or they assist them to get access to food". (Aboriginal LHD staff member)

Participants also explained how the unique kinship networks and connection with the wider Aboriginal community meant that Sorry business (funerals and cultural practices and protocols associated with death) happens quite regularly. Consequently, families have lots of mob (family) staying with them, which is often unplanned making it hard to budget and prepare for as "you don't know how many people are going to turn up to your door for a feed", an Aboriginal mother explained. In some instances, mob might stay for only a few nights or sometimes for months, which increases both parties' financial stress. Participants felt that government payments and food relief agency funding fail to take this into consideration, leaving many families further disadvantaged. However, a participant explained:

"...the AMS [Aboriginal Medical Service] will then try to assist through its various programs. If it is Sorry Business - and we know a lot of people are going to that house, then we will assist by dropping off tea, coffee, stuff like this, some items that keep them going whilst they've got a houseful". (ACCHS staff member)

"... well you know for an example, this morning, a community member just passed on. That means that the whole community will come together that are linked with this family, because of the stresses that the family are under now, the wider the community comes together to be able to provide those meals for those families". (ACCHS staff member)

\section{Fulfilling other family responsibilities}

Participants felt families are increasingly busy with children's after school activities which left less time for cooking meals at home. They described how the time pressures families face are driving them towards looking for quick and easy options for meals:

"So families with young kids that do sports and have extra curricular activities, a lot. Because by the time school's done and you're running to sports and what not, it's about 7:30, eight o'clock and it's easy to grab your big four. Macca's, KFC, Red Rooster and Hungry Jack's". (ACCHS staff member) 
"...some weeks they're really good and some weeks they're not really good. It just comes down to time again. Sometimes it's just easier to go and get some Macca's and let them eat it". (Aboriginal mother)

Participants explained that many Aboriginal grandparents, aunties, uncles and other family members are caring for additional children, particularly where social and emotional well-being issues were affecting the family. They felt this put financial strain on those family members, which was hindering their ability to afford enough food:

"... a lot of the time grandparents are doing it, and there are so many kids in the household that it's so hard to look after everybody and feed everybody, and with the depression and anxiety on top of that, and sometimes drug addictions and things like that, they're just [keeping everyone alive]". (Aboriginal Education staff member)

\section{Recommendations for local action}

A number of potential solutions to help ease food insecurity in the two communities were suggested, including, introducing school breakfast programs, offering cooking and budgeting programs in the ACCHSs and schools, increased transport options, planting community vegetable gardens and establishing subsidised food boxes that cater to the communities' needs. Aboriginal community members also felt strongly that any food security programs should ensure that culture is at their heart. Participants suggested that culture needs to underpin any food security initiative, stating it would positively impact on participation, mental health, and overall wellbeing.

\section{Discussion}

This study gathered local Aboriginal community members and Aboriginal and non-Aboriginal stakeholders' perspectives on the drivers of food insecurity in two communities located in one urban area and one large regional area in NSW. Study participants reported that food insecurity was a huge problem experienced by the families in their communities on a regular basis. The key drivers of food insecurity described by the participants were consistent with other food security literature in Australia and internationally. These included, high rates of unemployment and low household income, inequities in the availability of affordable healthy food $(10,17,54-57)$, lack of transport options to purchase food $(54,56$, $58,59)$, distance to large supermarkets selling a greater variety of less expensive produce $(58,60-62)$, and an obesogenic environment $(55,56,59)$. Time pressures combined with a lack of nutrition knowledge $(63)$, cooking and budgeting skills drove families towards easy meal options (64-66). As found in other studies with Aboriginal people and other Indigenous peoples globally, unstable and inadequate housing and lack of home utilities, were issues impacting on families ability to be food secure $(55,67-69)$. In addition, community-relevant factors linked to colonization, cultural identity, racism and relationships with mainstream organizations further impacted on nutrition and food access in these settings $(59,69-71)$.

Financial barriers were described as a major factor impacting on participants food security and is commonly reported among Aboriginal communities $(16,22,54,55)$. The perceived high cost of healthy 
food reported in this study is consistent with other studies in Aboriginal communities $(54,55,72-74)$, however, most of these studies have been conducted in remote areas, with relatively little price data available from urban areas $(16,74,75)$. Food affordability is not only impacted by the price of food, but also by income, as highlighted in a recent study in a comparative regional area indicating that price alone is not the major factor and that income combines with other affordability factors (57). In that study they compared the average cost of the Victorian Healthy Food Basket (76) with the same food basket in the study location, and found that only $44 \%$ of stores had access to all items and $34 \%$ of a four person family's government income support payments went towards purchasing the basket (57). In addition, households on low incomes spend a greater proportion on food than those on higher incomes $(19,74)$ and those living in the most socio-economically disadvantaged areas being the most impacted $(16,77)$. Evidence in remote Aboriginal communities shows slightly higher levels of food unaffordability which supports the earlier findings of the 2019 study, this is still unaffordable for most households (78).

Charities and food relief programs can play an important role in aiding families on low incomes who are experiencing food insecurity $(57,79)$, however, feelings of shame and fear of judgement can prevent some families from accessing these services $(64,65,80)$. We would argue that these feelings are even stronger barriers amongst Aboriginal communities (81) as many people have experienced and continue to experience family members being removed at alarming rates (51). Understandably, fear was particularly noted when accessing non-Aboriginal food relief organizations, which has been described in literature as a major barrier for Aboriginal people in accessing mainstream health services more generally (82-84) and also amongst Indigenous peoples globally (85). A lack of compassion and understanding for the trauma and on-going racism Aboriginal people face when accessing mainstream services was also described, which is consistent with other studies amongst Indigenous populations $(85,86)$.

Local food systems for Aboriginal peoples have changed drastically in Australia due to European invasion, colonization and subsequent oppression. As a result of colonization, many Aboriginal people were forced off Country (traditional lands) and made to live on missions and reserves (see footnote) where they were not allowed to maintain a traditional diet. Mission and reserve life forced Aboriginal people to consume an energy dense, 'Western' diet, provided by food rations such as white flour, rice, poor quality meat, sugar, tea, alcohol and tobacco $(52,70)$. In our study, this was viewed to currently impact on food choices made by many Aboriginal families currently due to a change of taste from traditional foods, loss of traditional food sources and knowledge on how to locate and prepare them. Participants described this loss of traditional foods and the knowledge has led to many community members consuming 'new foods' which have now been embraced as 'Aboriginal cultural foods', even though they were not part of a traditional diet. They also described how initiatives run by the local ACCHSs to reduce the consumption of unhealthy food, by introducing healthier options, has been met with resistance as many people are very protective over these unhealthy foods, perceiving them to be 'Aboriginal cultural foods'. Aboriginal families also described disruption to family structures as a result of the Stolen Generations (51) and children currently being placed in out of home care to have greatly impacted on important nutrition knowledge and cooking skills being passed on to children. Nutrition research conducted with young people leaving the 
out-of-home care system showed that most were consuming a diet predominately of foods with low nutritional value (87).

A number of coping strategies such as rationing, skipping meals and buying cheap unhealthy food to fill kids up, are highlighted in this study and have also been found in other studies in both Aboriginal populations and other populations internationally $(55,80,88)$. The sharing of food and resources amongst family and the wider Aboriginal community was also described as a way families deal with an inadequate income and was viewed positively as an important cultural practice. Sharing as a cultural practice is an important part of continuing social connectedness amongst family and the wider Aboriginal community and has been discussed in other literature $(54,89,90)$ and was a key strategy used to deal with food insecurity in our study as stated in other studies on Aboriginal people $(54,66)$.

\section{Strengths and limitations}

A strength of this study is that participants were recruited through ACCHSs and not food relief organizations, which allows for a wider range of perspectives and not just of those accessing food assistance programs. Each interview had an Aboriginal researcher with at least 10 years' experience working in ACCHSs. This played a positive role in the communication of the interview questions and in establishing trusting relationships in which people felt comfortable to share personal experiences.

The following limitations should be considered in relation to the findings from this study. Firstly, the data were collected with two Aboriginal communities, one in an urban area and another in a large regional area of NSW. Aboriginal communities throughout Australia are diverse so the transferability of the findings to other populations and setting is uncertain. The findings from this study may resonate with other Aboriginal communities in urban and large regional areas, as there were similarities with other studies in similar settings. Secondly, although this study approached several stakeholders from the food supply industry, we only had one agree to participate in the project.

\section{Conclusion}

This study found that Aboriginal families in urban and regional areas are experiencing food insecurity on a regular basis, which is impacted by a range of socio-economic, environmental, systemic and cultural factors, as reported by the study participants. The study identified several contributing factors including poverty and unemployment, inequities in the availability and accessibility of affordable healthy food, and generational loss of healthy food knowledge. Further work is needed to explore several important areas impacting on Aboriginal food insecurity, such as the local food environment and food system as well as culture, traditional foods, and the on-going impacts of colonization that impact food choices and consumption, and Aboriginal food sovereignty. There is a need for partnerships to be strengthened between Aboriginal organizations, local government, food suppliers and the health sector and coordinated efforts to drive the co-design of recommended programs and policies to improve food security and health outcomes for Aboriginal people. 


\section{Abbreviations}

ACCHS = Aboriginal Community Controlled Health Service

NGO = Non-Governmental Organization

Vinnies $=$ St Vincent de Paul Society, a Catholic aid agency

LHD $=$ Local Health District

\section{Declarations}

\section{Ethics approval and consent to participate}

Ethics approval for this study was provided by the Aboriginal Health and Medical Research Council (AH\&MRC) of NSW Ethics Committee (1226/16). The research methods were carried out in accordance with the AH\&MRC of NSW Ethics Committee guidelines for research with Aboriginal peoples.

All participants were aged over 18 years and provided written, informed consent to participate in the research project.

\section{Consent for publication}

NA

Availability of data and materials

The qualitative data set used during the current study is available from the corresponding author on request.

\section{Competing interests}

The authors declare there are no competing interests.

\section{Funding}

This work was supported by The Australian Prevention Partnership Centre, funded through the National Health and Medical Research Council of Australia (NHMRC) Partnership Centre grant scheme (grant ID: GNT9100001) with the Australian Government Department of Health, the NSW Ministry of Health, ACT Health and the HCF Research Foundation. The work was also funded by grants from the NHMRC through the Study of Environment on Aboriginal Resilience and Child Health (SEARCH) (\#358457, \#1023998 and \#1035378). Simone Sherriff was supported by a Turner PhD scholarship from the Charles Perkins Centre, University of Sydney.

Authors' contributions 
SM and AL conceptualized the study and designed the interview guides. SS, SM, DK and JN conducted the interviews. SS, SM, DK and JN drafted the manuscript. NN and AT provided analytical support in the development of themes and reviewed the manuscript and provided comments. JN, SE, TI, KS, MD and AL reviewed the manuscript and provided comments.

\section{Acknowledgements}

'Murradambirra Dhangaang' in the title of this paper is in Wiradjuri language and means 'make food secure' and was a title determined by our ACCHS partners. We would like to acknowledge the Traditional Custodians 'the Wiradjuri and Tharawal peoples' of the lands in which this research was conducted and pay our respects to their Elders past and present. We would also like to thank all of the community members and stakeholders who participated in this project, without them this research would not have been possible.

\section{Footnote}

The term 'mission era' used by participants in this study refers to when Aboriginal peoples were forced to live on missions, managed reserves, and stations as formally set up spaces with the aim to control all aspects of Aboriginal people's lives. Missions were created by churches or religious individuals on land granted to them by the government to house Aboriginal people to train them in Christian ideals and work (91). Reserves were land set aside by the government for Aboriginal people to live on and some were 'unmanaged' and received rations such as rice, flour, sugar and occasionally fatty meats, and blankets. Stations or managed reserves were set up to have schooling for the preparation of the workforce, rations were given, and housing was often provided. Station managers would control who could or could not live on the station (91).

Prior to European settlement and the colonization of Australia, Aboriginal peoples maintained a healthy nutrient-dense diet for tens of thousands of years, but there has since been a transition in diets to ones that are unhealthy and energy-dense, which is predominately due to the rations received at missions and settlements and from the continued social and economic disadvantage Aboriginal people face in Australia $(53,90,92)$.

\section{References}

1. Australian Bureau of Statistics. Australian Aboriginal and Torres Strait Islander Health Survey: Nutrition Results - Food and Nutrients, 2012-13. Canberra, 2015.

2. World Bank. Food Security and COVID-19 Washington DC: The World Bank, 2021 [Available from: https://www.worldbank.org/en/topic/agriculture/brief/food-security-and-covid-19.

3. Kent K, Murray S, Penrose B, Auckland S, Visentin D, Godrich S, et al. Prevalence and sociodemographic predictors of food insecurity in Australia during the COVID-19 pandemic. Nutrients. 2020,12(9):2682. 
4. O'Kane G. COVID-19 puts the spotlight on food insecurity in rural and remote Australia. The Australian Journal of Rural Health. 2020,28(3):319.

5. Food and Agriculture Organization of the United Nations. Declaration of the world summit on food security. Rome, Italy FAO, 2009.

6. Burns $C$. A review of the literature describing the link between poverty, food insecurity and obesity with specific reference to Australia. Melbourne: VicHealth, 2004.

7. Huang J, Oshima KM, Kim Y. Does food insecurity affect parental characteristics and child behavior? Testing mediation effects. The Social Services Review. 2010,84(3):381-401.

8. Booth S, Smith A. Food security and poverty in Australia-challenges for dietitians. Australian Journal of Nutrition and Dietetics. 2001,58(3):150-6.

9. Cook JT, Frank DA, Levenson SM, Neault NB, Heeren TC, Black MM, et al. Child food insecurity increases risks posed by household food insecurity to young children's health. The Journal of nutrition. 2006,136(4):1073-6.

10. Turrell G, Kavanagh A. Socio-economic pathways to diet: modelling the association between socioeconomic position and food purchasing behaviour. Public Health Nutrition. 2005,9(3):375-83.

11. Seligman HK, Laraia BA, Kushel MB. Food insecurity is associated with chronic disease among lowincome NHANES participants. The Journal of nutrition. 2010,140(2):304-10.

12. National Health and Medical Research Council. Australian Dietary Guidelines. Canberra: National Health and Medical Research Council, 2013.

13. Australian Insitute of Health and Welfare. The health and welfare of Australia's Aboriginal and Torres Strait Islander peoples 2015. Canberra: Australian Institute of Health and Welfare, 2015. Report No.: Cat. no. IHW 147.

14. Australian Bureau of Statistics. Australian Aboriginal and Torres Strait Islander Health Survey: Consumption of Food Groups from the Australian Dietary Guidelines, 2012-13. Canberra, 2016.

15. Australian Institute of Health and Welfare. Australian Burden of Disease Study: Impact and causes of illness and death in Aboriginal and Torres Strait Islander people 2011. Canberra, 2016. Contract No.: Cat. no. BOD 7.

16. Lee A, Ride K. Review of nutrition among Aboriginal and Torres Strait Islander people. Australian Indigenous Health Bulletin. 2018.

17. Barosh L, Friel S, Engelhardt K, Chan L. The cost of a healthy and sustainable diet-who can afford it? Australian and New Zealand Journal of Public Health. 2014,38(1):7-12.

18. Harrison M, Lee A, Findlay M, Nicholls R, Leonard D, Martin C. The increasing cost of healthy food. Australian and New Zealand Journal of Public Health. 2010,34(2):179-86.

19. Kettings C, Sinclair AJ, Voevodin M. A healthy diet consistent with Australian health recommendations is too expensive for welfare-dependent families. Australian and New Zealand Journal of Public Health. 2009,33(6):566-72.

20. Friel S, Pescud M, Malbon E, Lee A, Carter R, Greenfield J, et al. Using systems science to understand the determinants of inequities in healthy eating. PLoS One. 2017,12(11):e0188872. 
21. Saethre E. Nutrition, Economics and Food Distribution in an Australian Aboriginal Community. Anthropological Forum. 2005,15(2):151-69.

22. Lee A, Ride K. Review of programs and services to improve Aboriginal and Torres Strait Islander nutrition and food security. Australian Indigenous Health Bulletin. 2018,1:3.

23. Astell-Burt T, Feng X. Geographic inequity in healthy food environment and type 2 diabetes: can we please turn off the tap? Medical Journal of Australia. 2015,203(6):246-8.e1.

24. Whelan J, Millar L, Bell C, Russell C, Grainger F, Allender S, et al. You can't find healthy food in the bush: poor accessibility, availability and adequacy of food in rural Australia. International Journal of Environmental Research and Public Health. 2018,15(10):2316.

25. Bardenhagen CJ, Pinard CA, Pirog R, Yaroch AL. Characterizing rural food access in remote areas. Journal of Community Health. 2017,42(5):1008-19.

26. Turrell G, Bentley R, Thomas LR, Jolley D, Subramanian S, Kavanagh AM. A multilevel study of area socio-economic status and food purchasing behaviour. Public Health Nutrition. 2009,12(11):2074-83.

27. Global Panel on Agriculture and Food Systems for Nutrition. Food systems and diets: Facing the challenges of the 21st century. London, 2016.

28. Halliday J, Platenkamp L, Nicolarea Y. A menu of actions to shape urban food environments for improved nutrition, GAIN, MUFPP and RUAF. 2019.

29. Ruel M, Garrett J.L, Yosef S. 'Growing Cities, New Challenges.' in International Food Policy Research Institute (IFPRI) Global Food Policy Report. Washington, DC, 2017.

30. Dixon J, Omwega AM, Friel S, Burns C, Donati K, Carlisle R. The health equity dimensions of urban food systems. Journal of Urban Health. 2007,84(1):118-29.

31. Bramwell L, Foley W, Shaw T. Putting urban Aboriginal and Torres Strait Islander food insecurity on the agenda. Australian journal of primary health. 2017,23(5):415-9.

32. Committee on a Framework for Assessing the Health E, Social Effects of the Food System, Food and Nutrition Board, Board on Agriculture and Natural Resources, Institute of Medicine, Council. NR. A framework for assessing effects of the food system. Nesheim MC, Oria M, Yih PT, editors. Washington (DC): The National Academies Press, 2015.

33. Signal LN, Walton MD, Ni Mhurchu C, Maddison R, Bowers SG, Carter KN, et al. Tackling 'wicked' health promotion problems: a New Zealand case study. Health Promotion International. 2013,28(1):84-94.

34. Hernández A, Ruano AL, Marchal B, San Sebastián M, Flores W. Engaging with complexity to improve the health of indigenous people: a call for the use of systems thinking to tackle health inequity. International Journal for Equity in Health. 2017,16(1):1-5.

35. Panaretto KS, Wenitong M, Button S, Ring IT. Aboriginal community controlled health services: leading the way in primary care. Medical Journal of Australia. 2014,200(11):649-52.

36. Foley D. Indigenous epistemology and Indigenous standpoint theory. Social alternatives. 2003,22(1):44. 
37. National Aboriginal Community Controlled Health Organisation (NACCHO). Aboriginal health: definitions 2018 [Available from: www.naccho.org.au/about/aboriginal-health/definitions.

38. Australian Bureau of Statistics. 2016 Census QuickStats 2016 [Available from: https://quickstats.censusdata.abs.gov.au/census_services/getproduct/census/2016/quickstat/1034.

39. Australian Bureau of Statistics. 2016 Census QuickStats 2016 [Available from: https://quickstats.censusdata.abs.gov.au/census_services/getproduct/census/2016/quickstat/036.

40. SEARCH Investigators. The Study of Environment on Aboriginal Resilience and Child Health (SEARCH): study protocol. BMC Public Health. 2010,10(1):287.

41. Wright D, Gordon R, Carr D, Craig J, Banks E, Muthayya S, et al. The Study of Environment on Aboriginal Resilience and Child Health (SEARCH): a long-term platform for closing the gap. Public Health Research and Practice. 2016.

42. Sherriff SL, Miller H, Tong A, Williamson A, Muthayya S, Redman S, et al. Building trust and sharing power for co-creation in Aboriginal health research: a stakeholder interview study. Evidence \& Policy: A Journal of Research, Debate and Practice. 2019,15(3):371-92.

43. Miller HM, Young C, Nixon J, Talbot-McDonnell M, Cutmore M, Tong A, et al. Parents' and carers' views on factors contributing to the health and wellbeing of urban Aboriginal children. Aust N Z J Public Health. 2020,44(4):265-70.

44. Bowen GA. Grounded theory and sensitizing concepts. International journal of qualitative methods. 2006,5(3):12-23.

45. Rychetnik L, Webb K, Story L, Katz T. Food Security Options Paper: A planning framework and menu of options for policy and practice interventions. 2003.

46. Given LM. 100 questions (and answers) about qualitative research: SAGE Publications, 2015.

47. Corbin J, Strauss A. Basics of qualitative research: techniques and procedures for developing grounded theory,: Sage Publications, 2014.

48. Sandelowski M. Whatever happened to qualitative description? Research in nursing \& health. 2000,23(4):334-40.

49. Tong A, Sainsbury P, Craig J. Consolidated criteria for reporting qualitative research (COREQ): a 32item checklist for interviews and focus groups. International Journal for Quality in Health Care. 2007,19(6):349-57.

50. The Healing Foundation. Who are the stolen generations? n.d [Available from: https://healingfoundation.org.au/resources/who-are-the-stolen-generations/.

51. Family Matters, SNAICC, University of Melbourne, Monash University, Griffith University. The Family Matters Report 2019: Measuring trends to turn the tide on the over-representation of Aboriginal and Torres Strait Islander children in out-of-home care in Australia. Melbourne, Victoria, 2019.

52. Shannon C. Acculturation: Aboriginal and Torres Strait Islander nutrition. Asia Pacific Journal of Clinical Nutrition. 2002,11:S576-S8.

53. Brimblecombe J, Maypilama E, Colles S, Scarlett M, Dhurrkay JG, Ritchie J, et al. Factors influencing food choice in an Australian Aboriginal community. Qualitative health research. 2014,24(3):387-400. 
54. McCarthy L, Chang AB, Brimblecombe J. Food security experiences of Aboriginal and Torres Strait Islander families with young children in an urban setting: influencing factors and coping strategies. International Journal of Environmental Research and Public Health. 2018,15(12):2649.

55. Thorpe S, Browne J. Closing the Nutrition \& Physical Activity Gap in Victoria: Victorian Aboriginal Nutrition \& Physical Activity Strategy: Policy Proposals for Promoting Healthy Eating and Physical Activity Among Indigenous Australians, 2009-2014: Victorian Aboriginal Community Controlled Health Organisation, 2009.

56. Crawford B, Byun R, Sainsbury P. The relocation of public housing tenants in southwestern Sydney: A health impact assessment. Sydney: Population Health, South Western Sydney Local Health District. 2015.

57. Mungai Nw, Priestly J, Pawar M. Food insecurity in regional rural Australia. Australian Social Work. 2020,73(2):149-61.

58. Turrell G, Hewitt B, Patterson C, Oldenburg B, Gould T. Socioeconomic differences in food purchasing behaviour and suggested implications for diet-related health promotion. Journal of Human Nutrition and Dietetics. 2002,15(5):355-64.

59. Palermo C, Mitchell C. Urban Victorian Aboriginal Community Nutrition Needs Assessment Report. 2002. Victorian Aboriginal Health Service: Melbourne.

60. Sooman A, Macintyre S, Anderson A. Scotland's health-a more difficult challenge for some? The price and availability of healthy foods in socially contrasting localities in the west of Scotland. Health bulletin. 1993,51(5):276-84.

61. Curtis KA, McClellan S. Falling through the safety net: poverty, food assistance and shopping constraints in an American city. Urban Anthropology and Studies of Cultural Systems and World Economic Development. 1995:93-135.

62. Ellaway A, Macintyre S. Shopping for food in socially contrasting localities. British food journal. 2000,102(1):52-9.

63. Jabs J, Devine CM. Time scarcity and food choices: an overview. Appetite. 2006,47(2):196-204.

64. Garthwaite K. Stigma, shame and 'people like us': an ethnographic study of foodbank use in the UK. Journal of Poverty and Social Justice. 2016,24(3):277-89.

65. Pollard CM, Mackintosh B, Campbell C, Kerr D, Begley A, Jancey J, et al. Charitable food systems' capacity to address food insecurity: An Australian capital city audit. International Journal of Environmental Research and Public Health. 2018,15(6):1249.

66. Bhawra J, Cooke MJ, Hanning R, Wilk P, Gonneville SL. Community perspectives on food insecurity and obesity: focus groups with caregivers of Métis and off-reserve first nations children. International Journal for Equity in Health. 2015,14(1):96.

67. Kushel MB, Gupta R, Gee L, Haas JS. Housing instability and food insecurity as barriers to health care among low-income Americans. Journal of General Internal Medicine. 2006,21(1):71-7.

68. Victorian Aboriginal Community Controlled Health Organisation. Feeding Our Future: Aboriginal Early Childhood Nutrition \& Physical Activity Needs Assessment Report. Melbourne, Victoria, 2011. 
69. Baskin C, Guarisco B, Koleszar-Green R, Melanson N, Osawamick C. Struggles, strengths and solutions: Exploring food security with young urban Aboriginal moms. Esurio: Ontario Journal of Hunger and Poverty. 2009,1(1).

70. Browne J, Laurence S, Thorpe S. Acting on food insecurity in urban Aboriginal and Torres Strait Islander communities. Policy and practice interventions to improve local access and supply of nutritious food. 2009.

71. Reilly R, Doyle J, Rowley K. Koori community-directed health promotion in the Goulburn Valley. Australian Community Psychologist. 2007,19(1):39-46.

72. Abbott PA, Davison JE, Moore LF, Rubinstein R. Effective nutrition education for Aboriginal Australians: lessons from a diabetes cooking course. Journal of Nutrition Education and Behavior. 2012,44(1):55-9.

73. Ferguson M, O'Dea K, Chatfield M, Moodie M, Altman J, Brimblecombe J. The comparative cost of food and beverages at remote Indigenous communities, Northern Territory, Australia. Australian and New Zealand journal of public health. 2016,40(S1):S21-S6.

74. Lee $A$, Lewis M. Testing the price of healthy and current diets in remote Aboriginal communities to improve food security: development of the Aboriginal and Torres Strait Islander Healthy Diets ASAP (Australian Standardised Affordability and Pricing) methods. International Journal of Environmental Research and Public Health. 2018,15(12):2912.

75. Godrich SL, Davies CR, Darby J, Devine A. What are the determinants of food security among regional and remote Western Australian children? Australian and New Zealand journal of public health. 2017,41(2):172-7.

76. Palermo C, Wilson A. Development of a healthy food basket for Victoria. Australian and New Zealand Journal of Public Health. 2007,31(4):360-3.

77. Pollard C, Savage V, Landrigan T, Hanbury A, Kerr D. Food Access and Cost Survey. Perth: Department of Health, WA. 2015.

78. Markham F, Biddle N. Income, poverty and inequality. Canberra, ACT: Centre for Aboriginal Economic Policy Research (CAEPR), 2018.

79. McKay F, Lindberg R. The important role of charity in the welfare system for those who are food insecure. Australian and New Zealand Journal of Public Health. 2019,43(4):310-2.

80. Douglas F SJ, Kiezebrink K, Kyle J. Resourcefulness, desperation, shame, gratitude and powerlessness: Common themes emerging from a study of food bank use in Northeast Scotland. AIMS Public Health. 2015,2(3):297.

81. Hamelin A-M, Beaudry M, Habicht J-P. Characterization of household food insecurity in Quebec: food and feelings. Social Science \& Medicine. 2002,54(1):119-32.

82. Canuto K, Harfield S, Wittert G, Brown A. Listen, understand, collaborate: developing innovative strategies to improve health service utilisation by Aboriginal and Torres Strait Islander men. Australian New Zealand Journal of Public Health. 2019,43(4). 
83. Smith JA, Braunack-Mayer A, Wittert G, Warin M. " It's sort of like being a detective": Understanding how Australian men self-monitor their health prior to seeking help. BMC Health Services Research. 2008,8(1):56.

84. Australian Department of Health and Ageing. National Aboriginal and Torres Strait Islander Health Plan 2013-2023: Department of Health and Ageing, 2013.

85. Ontario Federation of Indigenous Friendship Centres. Food Security \& The Ontario Friendship Centres: A Discussion Paper. 2016.

86. Herring S, Spangaro J, Lauw M, McNamara L. The intersection of trauma, racism, and cultural competence in effective work with Aboriginal people: Waiting for trust. Australian Social Work. 2013,66(1):104-17.

87. Broad B, Saunders L. Involving young people leaving care as peer researchers in health research project: a learning experience. Research, Policy and Planning. 1998,16(1):1-9.

88. Eicher-Miller HA, Mason AC, Weaver CM, McCabe GP, Boushey CJ. Food insecurity is associated with iron deficiency anemia in US adolescents. The American Journal of Clinical Nutrition. 2009,90(5):1358-71.

89. Broome RL. Aboriginal Australians, black responses to white dominance 1788-2001. Crows Nest, NSW: Allen \& Unwin, 2002.

90. Brimblecombe JK. Enough for rations and a little bit extra. Darwin, NT: Institute of Advanced Studies, Charles Darwin University. 2007.

91. Perry LJ. 'Mission Impossible': Aboriginal survival before, during and after the Aboriginal Protection Era: University of Newcastle, 2014.

92. Lee A. The transition of Australian Aboriginal diet and nutritional health. World Review of Nutrition and Dietetics. 1996,79:1-52.

\section{Figures}




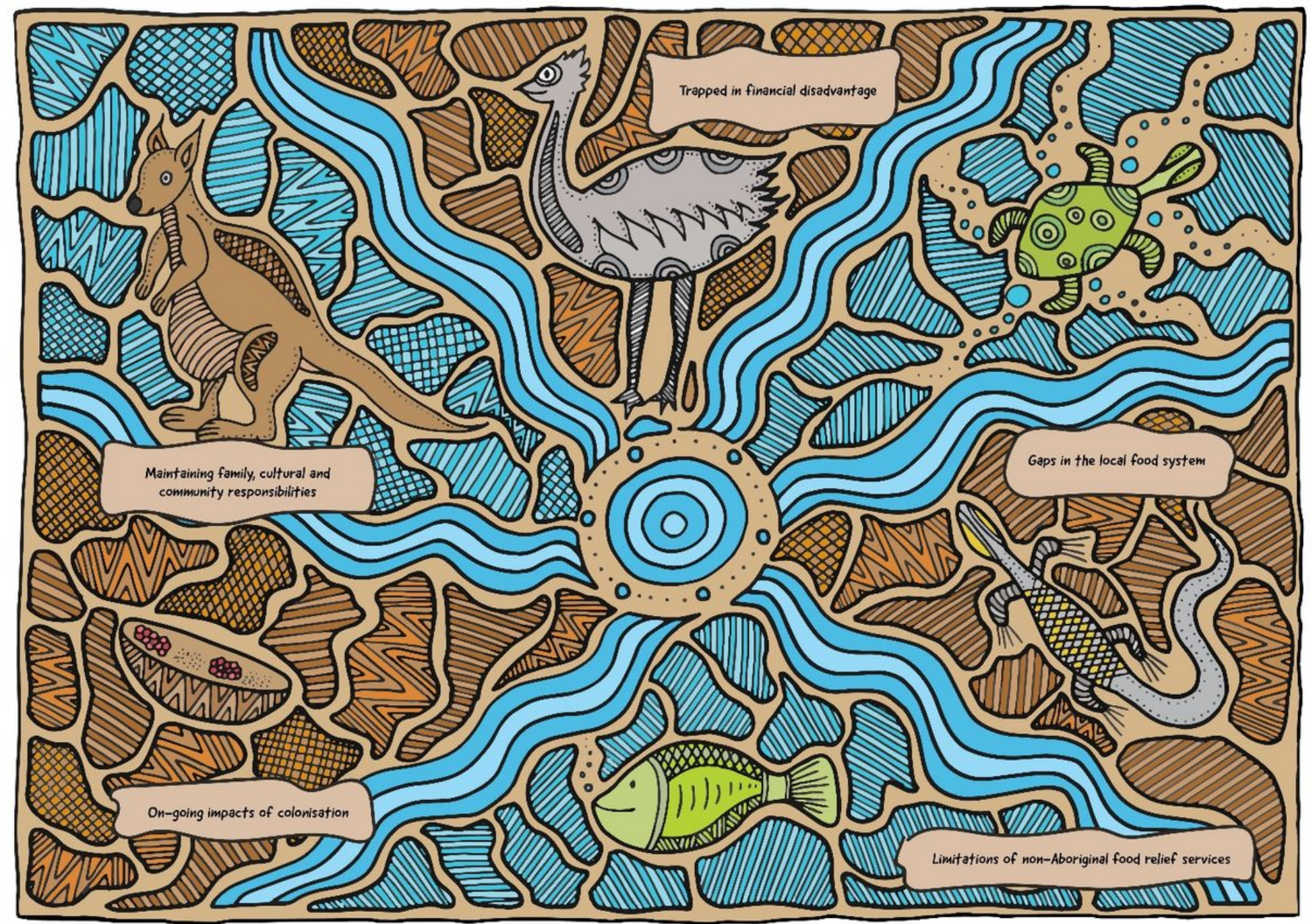

Figure 1

Key themes of food insecurity in two Aboriginal communities Artwork by Shakara Montalto at Deadly Designs, a proud Gunditjmara woman. Although this paper uses a Western written approach to tell a story of food insecurity in two Aboriginal communities, Aboriginal people have used song, dance, and art to tell our stories for many generations. The artwork in Figure 1 engages with a culturally grounded way of communicating that uses an Aboriginal lens to help position the paper within an Aboriginal context. The browns and orange patterns represent the land, and the strong connection Aboriginal people have to Country (traditional lands), and the blue swirls and circles represent the rivers and water ways that sweep throughout Australia. The bush tucker (traditional, native foods) in the artwork are the Kangaroo, Emu, Turtle, Goanna, Fish, and a Coolamon (curved piece of wood for collecting nuts, plants, seeds and carrying babies).

\section{Supplementary Files}

This is a list of supplementary files associated with this preprint. Click to download. 
- Supplementaryfile1.docx

Page 31/31 